
allemande

48-2 | 2016

Les espaces publics des pays germanophones, des espaces publics transnationaux?

\title{
Das Weimarer Dreieck: ein transnationales Thema in der deutschen und der polnischen Öffentlichkeit
}

\section{Rafał Ulatowski}

\section{(2) OpenEdition}

\section{Journals}

Édition électronique

URL : https://journals.openedition.org/allemagne/429

DOI : 10.4000 /allemagne.429

ISSN : 2605-7913

Éditeur

Société d'études allemandes

Édition imprimée

Date de publication : 28 décembre 2016

Pagination : $357-370$

ISSN : 0035-0974

Référence électronique

Rafał Ulatowski, „Das Weimarer Dreieck: ein transnationales Thema in der deutschen und der polnischen Öffentlichkeit", Revue d'Allemagne et des pays de langue allemande [Online], 48-2 | 2016, Online erschienen am: 28 Dezember 2017, abgerufen am 19 Mai 2021. URL: http:// journals.openedition.org/allemagne/429 ; DOI: https://doi.org/10.4000/allemagne.429 


\title{
Das Weimarer Dreieck: ein transnationales Thema in der deutschen und der polnischen Öffentlichkeit *
}

\author{
- Rafał Ulatowski**
}

Mit dem Ende des Kalten Krieges wurde ein neues Kapitel in der europäischen Geschichte aufgeschlagen. Die mittel- und osteuropäischen (MOE) Länder, zuerst Polen und Ungarn, signalisierten ihren Willen zur Annäherung an die Institutionen Westeuropas. Schon im Herbst 1989 wurden die ersten Schritte unternommen, damit sich die zwei Teile des geteilten Kontinents annähern ${ }^{(1)}$.

Das Ende des Kalten Krieges und die Wiedervereinigung haben die internationale Bedeutung Deutschlands aufgewertet. Obwohl Änderungen in der deutschen Außenpolitik befürchtet wurden ${ }^{(2)}$, zeichnete sich diese durch eine außergewöhnliche Kontinuität aus. Peter Katzenstein bezeichnete Deutschland als „gezähmte Macht“(3). Deutschland sollte in den internationalen Beziehungen nur multilateral aktiv sein. Die Europäische Union, die NATO und die anderen internationalen Organisationen und Institutionen sollten die deutsche Macht abmildern ${ }^{(4)}$. Deutschland war bemüht, das „regionale Milieu“ fortzuentwickeln. Die Vertiefung und Erweiterung der Europäischen Union standen im Mittelpunkt ${ }^{(5)}$. Um diese Ziele zu erreichen, setzte Deutsch-

* Dieser Beitrag entstand in Rahmen eines Aufenthalts an der Universität Straßburg, der durch ein BGF Forschungsstipendium der französischen Regierung gefördert wurde.

** Maître de conférences à l'Université de Varsovie, Institut des relations internationales.

1 Vgl. Frank Schimmelpfenning, Ulrich Sedelmeier (Hg.), The Europeanization of Central and Eastern Europe, Ithaca, Cornell University Press, 2005; Heather GrabBe, The EU's Transformative Power: Europeanization through Conditionality in Central and Eastern Europe, Houndmills, Palgrave Macmillan, 2006.

2 Vgl. Daniel Hamilton, „A More European Germany“, Journal of International Affairs, 45/1 (1991), S. 127-149.

3 Peter J. Katzenstein (Hg.), Tamed Power. Germany in Europe, Ithaca, Cornell University Press, 1998.

4 Vgl. Peter J. Katzenstein, „United Germany in an integrated Europe“, in: P. J. Katzenstein (Hg.), Tamed Power, ebd., S. 4.

5 Vgl. Simon Bulmer, Charlie Jeffery, William E. Paterson (Hg.), Germany's European Diplomacy: Shaping the Regional Milieu, Manchester, Manchester University Press, 2000; vgl. Peter 
land nicht nur auf die Kontinuität seiner engen Beziehungen mit Frankreich, sondern auch auf eine Verständigung mit Polen. Deutschland sollte mit befreundeten Staaten umgeben sein und aus dem Rand ins Zentrum Europas rücken.

$\mathrm{Zu}$ diesem Zweck wurde das europäische Projekt von Deutschland vorangebracht, das mit seinen beiden größten Nachbarn das Weimarer Dreieck gründete. Es sollte als Konsultationsforum der deutschen, französischen und polnischen Regierungen dienen und die drei Gesellschaften einander näher bringen ${ }^{(6)}$. Ziel dieses Beitrages ist, die Erfolge auf diesem Weg zu überprüfen. Es wird die These formuliert, das Weimarer Dreieck sei ein Elitenprojekt geblieben, ohne große Resonanz in der deutschen, französischen oder polnischen Öffentlichkeit ${ }^{(7)}$. Als Maßstab wird die deutsch-französisch-polnische Kooperation im Bereich Jugendaustausch und Hochschulwesen herangezogen. In diesem Beitrag wird der Sprachförderung eine besondere Aufmerksamkeit geschenkt, denn unsere Analyse soll die Entstehungsperspektiven der trilateralen deutsch-französisch-polnischen Öffentlichkeit erörtern, für die eine gemeinsame Sprache unerlässlich zu sein scheint ${ }^{(8)}$. Dieser Beitrag besteht aus vier Teilen: Zunächst werden die deutsch-französische Annäherung in der Nachkriegszeit und die deutschpolnische Annäherung in den 1990er Jahren kurz diskutiert. Im zweiten Teil werden die Entstehung, die Ziele, und die Erfolge der trilateralen Kooperation diskutiert. Im dritten Teil wird die Kooperation im Bereich Jugendaustausch und im vierten Teil im Bereich Hochschulwesen analysiert.

\section{Deutsch-französische und deutsch-polnische Annäherung}

Die bedingungslose Kapitulation Deutschlands im Mai 1945, die militärische Besetzung des Landes und seine Teilung in vier Zonen hatten die Abhängigkeit Deutschlands von den Großmächten zementiert. Mit dem Ausbruch des Kalten Krieges taten sich neue Chancen für Deutschland auf. Einerseits wurde die neue Bundesrepublik Deutschland von den Westalliierten (teilweise un-)abhängig, andererseits war die Regierung Konrad Adenauers davon überzeugt, die enge Verflechtung mit den USA, Großbritannien und Frankreich stelle eine Chance für Deutschland dar. Als überzeugter Europäer legte Adenauer die Grundlagen für die Westbindung der BRD

J. Katzenstein, A World of Regions Asia and Europe in the American Imperium, Ithaca, Cornell University Press 2005.

6 Ludger Kühnhardt, Henri Ménudier, Janusz Reiter, Das Weimarer Dreieck. Die französischdeutsch-polnischen Beziehungen als Motor der Europäischen Integration, Bonn, ZEI Discussion Paper, 2000.

7 Claudio Franzius, Ulrich K. Preuss (Hg.), Europäische Öffentlichkeit, Baden-Baden, Nomos, 2004; Michael BrüGGEMANn, Europäische Öffentlichkeit durch Öffentlichkeitsarbeit? Die Informationspolitik der Europäischen Kommission, Wiesbaden, VS, Verl. für Sozialwiss., 2008; Silke AdAm, Symbolische Netzwerke in Europa der Einfluss der nationalen Ebene auf europäische Öffentlichkeit. Deutschland und Frankreich im Vergleich, Köln, Halem, 2007.

8 Thomas Risse, „Zur Debatte um die (Nicht-) Existenz einer europäischen Öffentlichkeit“, Berliner Debatte Initial, 13/5-6 (2002), S. 15-23; Peter Graf KielmansegG, „Integration und Demokratie“, in: Markus Jachtenfuchs, Beate Kohler-Косн (Hg.), Europäische Integration, Opladen, Leske \& Budrich, 1996, S. 47-71; Dieter Grimm, Braucht Europa eine Verfassung?, München, Carl Friedrich von Siemens Stiftung, 1995; vgl. Karl Deutsch, Nationalism and Social Communication: An Inquiry into the Foundations of Nationality, Cambridge, MIT Press, 1953. 
(Im Anfang war Adenauer ${ }^{(9)}$ ), darin folgte er aber auch dem Wunsch der Alliierten, die BRD in die Institutionen des Westblocks zu integrieren („Im Anfang waren die Alliierten“(10)). Bundeskanzler Adenauer legte besonders großen Wert auf die Qualität der Beziehungen mit Frankreich. Anders als die als rationales Kalkül gedachten guten Beziehungen mit den USA waren ihm die Beziehungen mit Frankreich eine Herzensfrage. Westdeutsche und französische Entscheidungsträger waren gleichermaßen überzeugt, dass angesichts der neuen internationalen Lage die europäische Integration für beide Staaten eine Chance darstellt ${ }^{(11)}$, und dies erwies sich als voller Erfolg. Eine Vertiefung und Erweiterung der Europäischen Gemeinschaft erfolgte in den nächsten Jahrzehnten. Das Ziel war eine politische Union. Kleine Schritte in diese Richtung sollten zugleich Europa umbauen und die zwischenstaatliche Rivalität durch eine gegenseitig vorteilhafte Kooperation ersetzen ${ }^{(12)}$. Dabei wurden die BRD und Frankreich zur treibenden Kraft der europäischen Integration ${ }^{(13)}$.

Schon in den 1950er Jahren hatte Adenauer enge Verbindungen zu den französischen Politikern Pierre Mendès France und Guy Mollet aufgebaut. Als 1958 General Charles de Gaulle die Macht in Frankreich übernahm, folgte eine kurze Phase der Verschlechterung der gegenseitigen Beziehungen, die aber schnell während des Gipfels in Rambouillet im Juni 1960 endete. Adenauer und de Gaulle waren gewillt, die deutsch-französischen Beziehungen langfristig zu verbessern und unterschrieben in diesem Sinn den Élysée-Vertrag vom 22. Januar 1963. In den nächsten Jahrzehnten wuchs dieser Vertrag zum Symbol der deutsch-französischen Freundschaft, so dass die deutsch-französischen Beziehungen trotz immer wiederkehrender Höhen und Tiefen als Modell präsentiert wurden ${ }^{(14)}$.

Es war also keine ganze Überraschung, dass Bundeskanzler Helmut Kohl während seiner Visite in Warschau im November 1989 die deutsch-französischen Beziehungen der polnischen Seite als ein Modell der bilateralen Beziehungen schmackhaft machen wollte. Er formulierte damit eine klare und ambitionierte Perspektive für die deutsch-polnischen Beziehungen. Während des Gesprächs mit dem polnischen

9 Arnulf BARING, Im Anfang war Adenauer: die Entstehung der Kanzlerdemokratie, München, Deutscher Taschenbuch Verlag, 1982 (2. Aufl.).

10 Helga Haftendorn, „Im Anfang waren die Alliierten. Die alliierten Vorbehaltsrechte als Rahmenbedingung des außenpolitischen Handelns der Bundesrepublik Deutschland“, in: Hans-Hermann Hartwich, Göttrik Wewer (Hg.), Regieren in der Bundesrepublik V, Opladen, VS Verlag für Sozialwissenschaften, 1993, S. 41-92.

11 Vgl. Andrew Hurrell, „Regionalism in Theoretical Perspective“, in: Louise Fawcett, Andrew HurRELL (Hg.), Regionalism in World Politics. Regional Organization and International Order, Oxford, 1995, S. 47.

12 Vgl. Gideon Rachman, „Europe's Zero-Sum Dilemma“, The National Interest, May/June 2012, S. 43.

13 Vgl. Ulrike Guérot, Mark LeOnARD, The new German question: How Europe can get the Germany it needs, London, ECFR, 2011, S. 4.

14 Vgl. Ulrich Pfeil, „Zur Bedeutung des Élysée-Vertrags“, APuZ, 63/1-3 (2013), S. 3-8; Ulrich Krotz, Joachim SCHILD, Shaping Europe. France, Germany, and Embedded Bilateralism from the Elysée Treaty to Twenty-First Century Politics, Oxford, Oxford University Press 2012, S. 75-113; vgl. Lily Gardner Feldman, „The Principle and Practice of ,Reconciliation' in German Foreign Policy: Relations with France, Israel, Poland and the Czech Republic“, International Affairs (Royal Institute of International Affairs, 1944), 75/2 (1999), S. 333-356. 
Premierminister am 12.11.1989 erklärte er: „Europa braucht Polen, Polen braucht Europa. In diesem Europa brauchen auch Polen und Deutschland einander. Beide Länder sind Nachbarn - ob es uns gefällt oder nicht. Bekanntlich kann man sich seinen Nachbarn - ebenso wie die Mitglieder der Familie - nicht aussuchen. [...] Heute aber gilt es, ein neues, gutes Kapitel anzufangen. Ein ähnlich enges Verhältnis, wie wir es heute mit Frankreich unterhalten“(15). Trotz der guten Atmosphäre der Visite mussten zuerst die geopolitischen Fragen geklärt werden, zumal die Öffnung der Mauer in Berlin die Wiedervereinigung Deutschlands auf die Tagesordnung brachte. Die „2+4“-Verhandlungen zwischen den vier Großmächten und den zwei deutschen Staaten wurden mit dem „Vertrag [...] über die abschließende Regelung in Bezug auf Deutschland (Zwei-plus-Vier-Vertrag)“ beendet $^{(16)}$, der die Tür für die deutsche Wiedervereinigung öffnete und endlich wurde am 14. November 1990 mit dem „Vertrag zwischen der Bundesrepublik Deutschland und der Republik Polen über die Bestätigung der zwischen ihnen bestehenden Grenze“(17) die polnische Westgrenze bestätigt. Am 17. Juni 1991 unterzeichneten beide Staaten den „Vertrag [...] über gute Nachbarschaft und freundschaftliche Zusammenarbeit “(18), in dem sie sich verpflichteten, die polnischen Bemühungen um den Beitritt zur Europäischen Gemeinschaft zu unterstützen. In den europäischen Angelegenheiten waren die polnischen und die deutschen Interessen gleich. Deutschland, das in den polnischen Bemühungen um „Rückkehr nach Europa“(19) eine Schlüsselrolle spielte, befürwortete voll und ganz die Osterweiterung, die „die westeuropäische Stabilitätszone durch eine Erweiterung von EU und NATO nach Osten“ ausdehnen sollte ${ }^{(20)}$. Somit wurde die Qualität der Beziehungen mit Deutschland zur polnischen Staatsräson ${ }^{(21)}$. Der Beginn der 1990er Jahre war grundsätzlich von einer neuen Atmosphäre in den bilateralen Beziehungen charakterisiert und der Begriff „Versöhnung“ machte die Runde ${ }^{(22)}$.

15 Zit. nach: Wladyslaw Bartoszewski, „Angst vor der Großmacht? Deutschland und Polen nach dem Umbruch“, Internationale Politik, 55/9 (2000), S. 10.

16 Vertragüberdieabschließende Regelungin BezugaufDeutschlandvom 12.September 1990(„Zwei-plusVier-Vertrag“), Auswärtiges Amt, http://www.auswaertiges-amt.de/cae/servlet/contentblob/373162/ publicationFile/3828/ZweiPlusVier\%20\%28Text\%29.pdf.

17 Vertrag zwischen der Bundesrepublik Deutschland und der Republik Polen über die Bestätigung der zwischen ihnen bestehenden Grenze vom 14. November 1990, BGBl, 1991, II, S. 1329.

18 Vertrag zwischen der Bundesrepublik Deutschland und der Republik Polen über gute Nachbarschaft und freundschaftliche Zusammenarbeit vom 17. Juni 1991, in: Bulletin des Presse- und Informationsamtes der Bundesregierung vom 18. Juni 1991, Nr. 68, S. 541-546.

19 Vgl. Thomas Mehlyausen, Polens Rückkehr nach Europa: warum sich die Europäische Union für ihre Osterweiterung entschied, Berlin, LIT-Verlag, 2009.

20 Peter Scнмidт, „Deutsche Sicherheitspolitik im Rahmen von EU, WEU und NATO“, Außenpolitik, 47/3 (1996), S. 212.

21 Vgl. Anna Wolff-PowęSKA, „Polska racja stanu w procesie normalizacji stosunków z Niemcami w warunkach wolności i demokracji“, in: Witold M. Góralski (Hg.), Polska-Niemcy 1945-2007. Od konfrontacji do współpracy i partnerstwa w Europie, Warschau, PISM, 2007, S. 159-175.

22 Vgl. Dieter Bingen, „Einübung in erwachsene Partnerschaft. Die politischen Beziehungen zwischen Deutschland und Polen“, in: D. Bingen, Peter Oliver Loew, Krzysztof Ruchniewicz, Marek Zy BurA (Hg.), Erwachsene Nachbarschaft: die deutsch-polnischen Beziehungen 1991 bis 2011, Wiesbaden, Harrassowitz, 2011, S. 34; Klaus BACHMAnN, „Die Versöhnung muß von Polen ausgehen“, Die Tageszeitung, 05.08.1994, S. 12 . 


\section{Entstehung, Ziele und Erfolge des Weimarer Dreiecks}

Schon während des Kalten Krieges entwickelten sich die polnisch-französischen und die deutsch-polnischen Beziehungen dynamisch. Der politische und besonders der wirtschaftliche Austausch in den 70er Jahren des 20. Jahrhunderts erregten große Aufmerksamkeit. Auch in den 1980er Jahren verfolgten die Politiker in Bonn und Paris die Entwicklung der politisch-wirtschaftlichen Lage in Polen mit großer Aufmerksamkeit. Die Gründe dafür waren vielfältig und reichten von den Sorgen um die Stabilität Europas bis zur Frage nach der Zahlungsfähigkeit Polens. Deutschland und Frankreich, die zu den wichtigsten Gläubigern Polens gehörten, drängten seit Mitte 1989 auf eine Neugestaltung der Beziehungen mit Polen. Polen war ebenfalls an einer Verbesserung der bilateralen Beziehungen interessiert, denn es suchte die politischwirtschaftliche Annäherung mit den Ländern und Institutionen Westeuropas. Mit dem Regierungswechsel im Herbst 1989 wurde Polen zusammen mit Ungarn zum Vorreiter der Annäherung der postsozialistischen Staaten mit dem Westen ${ }^{(23)}$.

Der Fall der Berliner Mauer, die Wiedervereinigung Deutschlands und das Drängen der MOE-Staaten nach einer raschen Annäherung an den Westen wurden in Paris mit großer Vorsicht betrachtet. Als Alternative zur Osterweiterung der Europäischen Gemeinschaft schlug der französische Präsident François Mitterrand ein Projekt der Europäischen Konföderation vor, das sowohl in den deutschen als auch in den polnischen Regierungskreisen abgelehnt wurde ${ }^{(24)}$.

Als die Idee der Europäischen Konföderation während des Prager Konvents Mitte 1991 de facto begraben wurde, schlug der deutsche Außenminister Hans-Dietrich Genscher im Einvernehmen mit seinem französischen Amtskollegen, Außenminister Roland Dumas, und dem polnischen Außenminister Krzysztof Skubiszewski eine Dreieckskooperation vor. Diese sollte mehrere Funktionen erfüllen. Primär sollte sie Polen den Weg in die Europäische Gemeinschaft öffnen. Dann sollte sie Frankreich ermöglichen, seine Präsenz in Mitteleuropa zu stärken. Schließlich sollte sie auch Deutschland ermöglichen, das französische Interesse an Mitteleuropa zu fördern und die eigene Stärke in dieser Region hinter dem Deckmantel der trilateralen Kooperation zu verstecken ${ }^{(25)}$. Trotz des komplizierten Netzes unterschiedlicher Interessen war von Anfang an deutlich, dass die drei Spitzen des Weimarer Dreiecks nicht gleich einflussreich sind. Das Weimarer Dreieck war die Summe der deutsch-französischen Partnerschaft und der deutsch-polnischen Partnerschaft, wobei der deutsch-französische Bilateralismus den deutsch-polnischen Bilateralismus dominierte ${ }^{(26)}$. Seit dem

23 Vgl. Pierre-Frédéric Weber, „Das Weimarer Dreieck. Vom Gründungsoptimismus zur neuen Sinnsuche“, in: Dieter Bingen, Peter Oliver Loew, Krzysztof Ruchniewicz und Marek Zybura (Hg.), Erwachsene Nachbarschaft. Die deutsch-polnischen Beziehungen 1991 bis 2011, Wiesbaden, Harrassowitz, 2011, S. 77; vgl. Dariusz Jarosz, Maria PAsztor, Stosunki polsko-francuskie 1944-1980, Warschau, PISM, 2008; Dieter BINGEN, „Die Entwicklung der Beziehungen zwischen der Bundesrepublik Deutschland und Polen 1949-1990“, in: Wolf-Dieter EBerwein, Basil Kerski, Die deutsch-polnischen Beziehungen 1949-2000: Eine Werte- und Interessengemeinschaft?, Opladen, Leske+Budrich, 2001, S. 35-60. Vgl. P.-F. WeBer, „Das Weimarer Dreieck“ (Anm. 23), S. 80-81. Vgl. ebd., S. 81-82.

Vgl. Helena Ewa WyligaŁa, Trójkąt Weimarski: współpraca Polski, Francji i Niemiec w latach 19912004, Toruń, Wydawnictwo Adam Marszałek, 2010; Bogdan Koszel, Trójkąt Weimarski. Geneza. Działalność. Perspektywy Współpracy, Poznań, Instytut Zachodni, 2006, S. 210. 
polnischen EU-Beitritt befindet sich das Weimarer Dreieck auf der Suche nach einer neuen Identität ${ }^{(27)}$.

Auf das erste Treffen der drei Außenminister Frankreichs, Deutschlands und Polens am 28./29. August 1991 in Weimar ${ }^{(28)}$ folgte eine Reihe von Konsultationen zwischen den drei Partnern. Diese fanden nicht nur zwischen den Außenministern, sondern seit 1993 auch im Gipfelformat (Treffen der Staats- und Regierungschefs) statt. Der Austausch erfolgte auch auf der Ebene der Europa- und Fachminister. Auch die Parlamente und die Zivilgesellschaften wurden in die Kooperation einbezogen ${ }^{(29)}$.

Der erste noch inoffizielle Gipfel des Weimarer Dreiecks fand am 21. September 1991 in Gdańsk statt. Es gab sieben (offizielle und inoffizielle) Gipfel des Weimarer Dreiecks. Das letzte Treffen war im Jahre 2011 in Polen. Der große Kenner des Weimarer Dreiecks, Klaus-Heinrich Standke, weist darauf hin, dass fünf Besonderheiten das Gipfeltreffen des Weimarer Dreiecks auszeichneten. Erstens ist dies „das Engagement und persönliche Interesse der Teilnehmer an der Weiterführung der in der EU einzigartigen trilateralen Sonderbeziehung“. Zweitens ist „die stimmige ,Chemistry“ und das damit verbundene persönliche Vertrauen zwischen den drei Spitzenakteuren“. Drittens ist „die Kontinuität des sog. ,Geistes von Weimar“ bemerkenswert. Besonders der polnische Präsident Aleksander Kwaśniewski und sein französischer Amtskollege Jacques Chirac „haben immer wieder ihr persönliches Interesse an der trilateralen Zusammenarbeit betont und sichtbar ihr Vergnügen an dieser Art von Gipfelgesprächskultur gezeigt“. Viertens wurde das Weimarer Dreieck von den Politikern dazu benutzt, die eigene politische Position zu stärken, wie es das Beispiel des polnischen Staatspräsidenten Aleksander Kwasniewski belegt. Fünftens wurde das Gipfelformat trotz unterschiedlicher Verfassungslagen in den jeweiligen Ländern entwickelt. Drei Themengruppen wurden im Rahmen des Gipfelformats besonders intensiv betrachtet: Europapolitische Themen, die internationale politische Lage und die trilaterale Kooperation. In der dritten Themengruppe spielte die Bildung dauerhaft eine wichtige Rolle $^{(30)}$. Diese Entwicklung entspricht der Feststellung aus der „Gemeinsamen Erklärung“: „Wir wollen eine umfassende Politik der Zusammenarbeit in den Bereichen der Kultur, der Bildung, der Wissenschaft, der Medien und der Austauschprogramme. Es

27 Vgl. Krzysztof Miszczak, „Das ,Weimarer Dreieck' neu beleben“, Europäische Sicherheit, 53/6 (2004), S. 12-15; Georg Boomganden, Hat das ,Weimarer Dreieck' noch eine Zukunft? Herausforderungen für Politik und Kultur; Dokumentation des überarbeiteten Podiumsgesprächs, Darmstadt, DPI, 2007.

28 Gemeinsame Erklärung der Außenminister von Deutschland, Frankreich und Polen zur Zukunft Europas Weimar, 29. August 1991, in: Bulletin des Presse- und Informationsamts der Bundesregierung, Nr. 92 (1991), S. 735-737.

29 Vgl. „Weimarer Dreieck“, Auswärtiges Amt, 07.03.2016, http://www.auswaertiges-amt.de/DE/ Europa/Zusammenarbeit_Staaten/Weimarer-Dreieck_node.html (Zugriff am 15.03.2016); Krzysztof Miszczak, „Weimarer Dreieck a. D.?“, in: Heiner Timmermann, Helmut Wagner (Hg.), Die transatlantischen Beziehungen auf dem Prüfstand: Europa und die USA zwischen Bruch - Irritation Kooperation, Münster, Lit Verl., 2005, S. 128-133.

30 Klaus-Heinrich STANDKe, „Analytische Übersicht der 7 Gipfelgespräche“, in: Kl.-H. STANdKe (Hg.), Das Weimarer Dreieck in Europa, Thorn, Adam Marszalek-Verlag, 2010, S. 351-373; vgl. Emil PiETr AS, Katarzyna SochackA, „Trójkąt Weimarski - potencjalne dziedziny współpracy“, Biuletyn PISM, 27 (2003); Kai-Olaf LANG, „Gute Dienste und begrenzte Kooperation: Das Weimarer Dreieck im Zeichen europäischer Großkrisen“, WeltTrends, 23/110 (2015), S. 26-31. 
bleibt unser Bestreben, menschliche Begegnungen über Länder und Sprachgrenzen hinweg, wo immer möglich, zu fördern“(31).

\section{Jugendaustausch}

Kein Kooperationsgebiet erfreut sich eines größeren Interesses und öffentlicher Resonanz als der Jugendaustausch. Zwei Jugendwerke, das Deutsch-Französische Jugendwerk (DFJW) und das Deutsch-Polnische Jugendwerk (DPJW), prägen das Bild der Kooperation. Das im Jahre 1963 entstandene DFJW wurde als „das schönste Kind des Élysée-Vertrages“ bezeichnet. Das DPJW entstand dagegen im Kontext des „Vertrages zwischen der Bundesrepublik Deutschland und der Republik Polen über gute Nachbarschaft und freundschaftliche Zusammenarbeit“ von 1991. Am gleichen Tag wie der Freundschaftsvertrag wurde das Abkommen über die Gründung des DPJW ${ }^{(32)}$ unterschrieben. Obwohl die Entscheidung über die Gründung des DPJW im Jahre 1991 getroffen wurde, nahm es seine Arbeit erst im Jahre 1993 auf. Sein Aufbau folgte dem Beispiel des DFJW. Es ist festzustellen, dass sich der deutsch-französische und der deutsch-polnische Jugendaustausch gut entwickeln, dass auch trilaterale Projekte realisiert werden. Klaus-Heinrich Standke zeigt, dass in den 1990er Jahren und in der ersten Dekade des 21. Jahrhunderts durchschnittlich 600 polnische, 600 deutsche und 600 französische Jugendliche jährlich an den trilateralen Projekten teilgenommen haben. Im Jahre 2007 allein waren es 2500 Jugendliche, jeweils 1/3 aus Frankreich, 1/3 aus Deutschland und 1/3 aus Polen. Eine klare Schwäche präsentiert aber der französisch-polnische Jugendaustausch, für den genaue Zahlen leider nicht zugänglich sind ${ }^{(33)}$.

Die Kooperation der beiden Jugendwerke wurde mehrmals gewürdigt. Im Jahre 2007 wurde ihnen vom „Komitee für die Deutsch-Französisch-Polnische Zusammenarbeit“ der Adam-Mickiewicz-Preis verliehen. Im Mittelpunkt der Programme der beiden Jugendwerke stehen das Thema der europäischen Integration, der Erwerb interkultureller Kompetenzen und die Förderung europäischen Bewusstseins. Die Teilnehmer sollen sich als ein Teil Europas fühlen. Die persönlichen Erfahrungen aus der internationalen Zusammenarbeit sollen das Bewusstsein der jungen Menschen aus Frankreich, Deutschland und Polen prägen ${ }^{(34)}$.

Welche Früchte diese Bemühungen tragen, wird auf der Basis der statistischen Daten geprüft. Ein Schlüsselelement für die Annäherung und für bessere Verständigung zwischen den Völkern spielt die Sprache. Die beiden Jugendwerke legen deshalb großen Wert auf das Erlernen der Sprachen, oder wenigstens bemühen sie sich, das Interesse am Sprachenlernen anzureizen. Wie die Daten in den Tabellen 1 und 2 zeigen,

31 Gemeinsame Erklärung der Außenminister von Deutschland, Frankreich und Polen zur Zukunft Europas Weimar (Anm. 28).

32 Abkommen vom 17. Juni 1991 zwischen der Bundesrepublik Deutschland und der Republik Polen über das Deutsch-Polnische Jugendwerk, Bundesgesetzblatt, 1992, 2, S. 623-626.

33 Vgl. Klaus-Heinrich StandKe, „Jugendaustausch im Weimarer Dreieck“, in: Kl.-H. STANdKE (Hg.), Europa: Die deutsch-französisch-polnische Zusammenarbeit - Entstehung - Potentiale - Perspektiven, Thorn, Adam Marszalek Verlag, 2010, S. 609-611.

34 Vgl. Gerd Hoofe, „Die Rolle der Deutsch-Französischen und der Deutsch-Polnischen Jugendwerke im Rahmen des Weimarer Dreiecks“, in: Kl.-H. Standke (Hg.), Europa (Anm. 33), S. 613-617. 


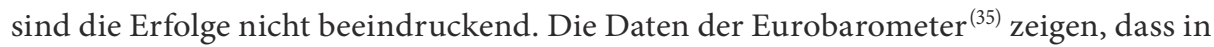
allen drei Staaten Englisch als die wichtigste Fremdsprache betrachtet wird. Während für die Bewohner Frankreichs Spanisch wichtiger als Deutsch ist, gehört Deutsch für die Bewohner Polens zu den zwei wichtigsten Fremdsprachen. Französisch dagegen befindet sich auf dem vierten Platz, hinter der russischen Sprache. Für die Bewohner Deutschlands hingegen ist Französisch die zweitwichtigste Fremdsprache.

Tabelle 1. Die zwei wichtigsten Fremdsprachen für persönliche Entwicklung und für die Karriere (Daten in \%)

\begin{tabular}{|c|c|c|c|c|c|c|}
\cline { 2 - 7 } \multicolumn{1}{c|}{} & Englisch & Französisch & Deutsch & Spanisch & Russisch & Italienisch \\
\hline Frankreich & 82 & 2 & 20 & 37 & 1 & 7 \\
\hline Polen & 72 & 5 & 46 & 2 & 9 & 1 \\
\hline Deutschland & 81 & 28 & 5 & 12 & 5 & 3 \\
\hline
\end{tabular}

Quelle: „Europeans and their Languages“, 2005, http://ec.europa.eu/public_opinion/archives/ebs/ebs_243_en.pdf, S. 32.

Tabelle 2. Zwei Fremdsprachen, die die Kinder lernen sollen (Daten in \%)

\begin{tabular}{|c|c|c|c|c|c|c|}
\cline { 2 - 7 } \multicolumn{1}{c|}{} & Englisch & Französisch & Deutsch & Spanisch & Russisch & Italienisch \\
\hline Frankreich & 91 & 2 & 24 & 45 & 0 & 6 \\
\hline Deutschland & 89 & 45 & 3 & 16 & 6 & 2 \\
\hline Polen & 90 & 7 & 69 & 1 & 10 & 1 \\
\hline
\end{tabular}

Quelle: „Europeans and their Languages“, 2005, http://ec.europa.eu/public_opinion/archives/ebs/ebs_243_en.pdf

Die Daten sind ähnlich, wenn man nach den wichtigsten Fremdsprachen für die Kinder fragt. In diesem Fall wird die Nützlichkeit einer Sprache in der langen Perspektive betrachtet. Der Hauptunterschied ist die größere Zustimmung für English als die „wichtigste“ Fremdsprache. Es verwundert aber, dass in Frankreich nach über 50 Jahren Arbeit des DFJW nur zwischen 20\% und 24\% der Befragten die Bedeutung der deutschen Sprache schätzen. Die Überzeugung, dass die Kinder Spanisch lernen sollen, ist deutlich höher. Die Gründe dafür sind unterschiedlich. Die Untersuchungen zeigen, dass in Frankreich ein rationales Verhältnis zu Fremdsprachen herrscht und von der ersten Fremdsprache bestimmte Nützlichkeit erhofft wird. Während Englisch diese Bedürfnisse sehr gut erfüllt, sind Hoffnungen gegenüber der zweiten Fremdsprache anderer Natur. Diese Sprache soll eher einfach beim Erlernen sein. Auch die „Mode“ spielt bei der Wahl eine wichtige Rolle, und Deutsch gilt zurzeit als weder einfach noch modisch ${ }^{(36)}$.

Man sollte auch darauf hinweisen, dass die Daten ein mangelndes Interesse an der polnischen Sprache nahelegen. Das muss nach über zwanzig Jahren Arbeit des DPJW mit Enttäuschung betrachtet werden. Auf dieses Defizit wird seit langem hingewiesen. Um

35 Die Daten stammen von 2005. Die Umfrage wurde von 5. November bis 7. Dezember 2005 in 25 EU Mitgliedstaaten, in Bulgarien, Rumänien, Kroatien und in der Türkei durchgeführt. 28694 Personen nahmen in der Umfrage teil.

36 Tina Nestler, Die Situation der deutschen Sprache im schulischen Bildungswesen Frankreichs, Hamburg, Diplomica Verlag, 2004, S. 69-71. 
dies zu bekämpfen, wurden unterschiedliche Ideen entwickelt. Hier lohnt es sich, auf das Projekt des Deutschen Polen-Instituts (DPI) in Darmstadt aufmerksam zu machen. Das DPI realisiert seit dem Schuljahr 2015/2016 das PolenMobil-Projekt. Es hat zum Ziel, die polnische Sprache zu vermitteln, ohne jedoch auf dieses Ziel beschränkt zu sein. „Im Rahmen von Unterrichtsbesuchen sollen landeskundliche, historische, kulturelle und politische Inhalte zu Polen, die später auch im Unterricht aufgegriffen werden können, vermittelt werden und auf das Land neugierig machen. “ Dieses Angebot steht allen Schularten und -formen offen. Die Reaktionen sind positiv. Die Organisatoren bemühen sich, das Projekt für das Schuljahr 2016/2017 zu verlängern ${ }^{(37)}$.

Die Daten über Meinungen der EU-Bewohner zum Thema Nützlichkeit der einzelnen Sprachen zeigen, dass die Osterweiterung im Jahre 2004 zu großen Veränderungen geführt hat. Im Jahre 2001 haben EU-weit noch 40\% der Befragten Französisch als nützlich eingestuft, Deutsch dagegen nur 23\% der Befragten. Im Jahre 2005 wurde Französisch von 25\% der Befragten und Deutsch von $22 \%$ der Befragten als nützliche Fremdsprache betrachtet. Im Vergleich dazu betrachteten über 2/3 der Befragten Englisch als eine nützliche Fremdsprache ${ }^{(38)}$.

In diesem Kontext ist es interessant auf die Rede von Bundespräsident Joachim Gauck in Februar 2013 hinzuweisen. Er hat den Aufbau der europäischen Öffentlichkeit gefordert und Englisch in der Rolle der lingua franca gestellt ${ }^{(39)}$. Dieser Vorschlag ist gleichermaßen mutig wie nicht realisierbar. Obwohl Bundespräsident Gauck richtig darauf hingewiesen hat, dass die meisten EU-Bürger Englisch können oder wenigstens lernen, würde die Realisierung dieses Vorschlags gegen die bisherige EU-Politik verstoßen, das Interesse der einzelnen EU-Staaten bedrohen und das Demokratieprinzip beeinträchtigen ${ }^{(40)}$.

Tabelle 3. Zahl der Teilnehmer der/an DFJW-Programme in ausgewählten Jahren

\begin{tabular}{|l|r|}
\hline 2005 & 141350 \\
\hline 2010 & 211416 \\
\hline 2014 & 194043 \\
\hline
\end{tabular}

Quelle: DFJW, Tätigkeitsbericht 2014.
Tabelle 4. Aufteilung der Teilnehmer der DFJW-Programme nach Herkunftsland im Jahre 2014

\begin{tabular}{|c|c|c|}
\hline & Zahl & \% \\
\hline Deutschland & 101993 & 52,6 \\
\hline Frankreich & 89636 & 46,2 \\
\hline Drittland & 2414 & 1,2 \\
\hline
\end{tabular}

Quelle: DFJW, Tätigkeitsbericht 2014.

37 Das PolenMobil ist auf Tour! Polen in der Schule, http://www.poleninderschule.de/polenmobil/ (Zugriff am 28.03.2016).

38 Vgl. „Europeans and their Languages“, http://ec.europa.eu/public_opinion/archives/ebs/ebs_243_en.pdf, S. 30 .

39 Joachim GaUCK, „Rede zu Perspektiven der europäischen Idee. Europa: Vertrauen erneuern - Verbindlichkeit stärken“, 22.02.2013, http://www.bundespraesident.de/SharedDocs/Downloads/DE/ Reden/2013/02/130222-Europa.pdf?__blob=publicationFile (Zugriff am 15.05.2016).

40 Philip Oltermann, „Something in common: should English be the official language of the EU?“, The Guardian, 24.04.2013, https://www.theguardian.com/world/2013/apr/24/europa-english-officiallanguage-eu (Zugriff am 15.05.2016). Vgl. Juliane House, „English as a lingua franca: A threat to multilingualism?", Journal of Sociolinguistics, 7/4 (2003), S. 556-578; Robert PHillipson, English-Only Europe? Challenging Language Policy, Routledge, London 2003; Margie Berns, „English in the European Union“, English Today, 11/3 (1995), S. 3-11. 
Dass die deutsche und französische Regierung fest entschlossen sind, die Jugendlichen der beiden Staaten einander anzunähern, zeigen die statistischen Daten unmissverständlich. Zwischen 2005 und 2010 ist die Zahl der Teilnehmer der DFJWProgramme um 50\% gestiegen. Ca. 200000 Jugendliche nehmen jährlich an den unterschiedlichen Programmen des DFJW teil. Immer öfter nehmen auch polnische Jugendliche an den Programmen des DFJW teil. „Der Austausch mit Polen nimmt innerhalb der trilateralen Begegnungen weiterhin eine privilegierte Stellung ein“, stellt der DFJW-Tätigkeitsbericht 2014 fest $^{(41)}$.

Tabelle 5. Schulischer und außerschulischer Austausch Deutschland-Polen, Teilnehmerzahlen

\begin{tabular}{|r|r|r|r|r|}
\cline { 2 - 5 } \multicolumn{1}{c|}{} & \multicolumn{1}{c|}{1993} & $\mathbf{2 0 0 5}$ & $\mathbf{2 0 1 0}$ & \multicolumn{1}{c|}{2013} \\
\hline Teilnehmer (insgesamt) & 46400 & 165350 & 113455 & 109080 \\
\hline Außerschulischer Austausch & - & 89090 & 49872 & 44620 \\
\hline Schulischer Austausch & - & 76260 & 63583 & 64460 \\
\hline Aus Polen & 22500 & 80864 & 54658 & 52260 \\
\hline Aus Deutschland & 23900 & 80346 & 55718 & 53559 \\
\hline Aus einem Drittland & - & 4140 & 3079 & 3261 \\
\hline
\end{tabular}

Quelle: DPJW, Daten und Fakten 1993-2010, Potsdam/Warschau, Dezember 2011; DPJW, Daten und Fakten 2013, Potsdam/Warschau, Dezember 2014.

Die Dynamik der Teilnehmerzahl der Programme des DPJW präsentiert sich anders. Kurz nach dem polnischen EU-Beitritt nahmen an den Programmen des DPJW mehr Teilnehmer als an den Programmen des DFJW teil. Danach nahm die Zahl der Teilnehmer ab, wobei das Jahr 2005 für das DPJW ein besonderes Jahr darstellte. „Ein so auffälliger Anstieg des gegenseitigen Interesses junger Polen und Deutschen in diesem Zeitraum ist auf den Beitritt Polens zur Europäischen Union im Vorjahr zurückzuführen, was das Interesse Jugendlicher für das Nachbarland und seine Menschen aufs Neue weckte. Außerdem war das Jahr 2005 ein Jahr zahlreicher wichtiger Jubiläen für die deutsch-polnischen Beziehungen: Gefeiert wurde der 60 . Jahrestag der Beendigung des Zweiten Weltkriegs und die Befreiung des Konzentrationslagers in Auschwitz-Birkenau, sowie der 40. Jahrestag der Übersendung des Hirtenbriefes der polnischen Bischöfe an ihre deutschen Amtsbrüder. Darüber hinaus erklärten beide Regierungen das Jahr 2005 zum Deutsch-Polnischen Jahr, was zusätzlich zu einer Fülle an Jugendinitiativen auf beiden Seiten der Oder führte. Ein weiteres Ereignis, das direkt zum Anstieg der Zahl deutschpolnischer Treffen beitrug, waren die Weltjugendtage in Köln. Viele schulische Partnerschaften und Jugendorganisationen nutzten diese Gelegenheit, um im Rahmen eines gemeinsamen Projekts an dem Weltjugendtreffen teilzunehmen“(42). Auch im Jubiläumsjahr 2016 (25. Jubiläum des deutsch-polnischen Nachbarschaftsvertrags) kann man wachsendes Interesse an der DPJW Projekten beobachten. Schon im Mai 2016 wurden die Mittel ausge-

41 OFAJ/DFJW, Rapport d'activité de l'Office franco-allemand pour la Jeunesse / Tätigkeitsbericht des Deutsch-Französischen Jugendwerks, 2014, S. 28. 
schöpft $^{(43)}$. Heute beträgt die Zahl der Teilnehmer an den DPJW-Programmen knapp über 50\% der Teilnehmer an den DFJW-Programmen. Relativ häufig werden Projekte des DPJW mit französischen Partnern realisiert. Im Jahre 2013 war Frankreich mit 23 Projekten das dritt-wichtigste Drittland. Auf dem ersten Platz stand die Tschechische Republik (67 Projekte) und auf dem zweiten Platz die Ukraine (60 Projekte). Diese Reihenfolge entspricht dem langjährigen Trend ${ }^{(44)}$. Die deutsch-polnische und die deutsch-französische Jugendaustausch entwickeln sich sehr gut ${ }^{(45)}$.

Um die trilaterale Zusammenarbeit zu fördern, wurden zahlreiche Ideen entwickelt und Initiativen ergriffen. Sie reichen von einem trilateralen Festival, der Erweiterung der Aktivitäten der beiden Jugendwerke, der Entstehung eines neuen trilateralen Jugendwerks, der besseren Finanzierung der beiden Jugendwerke, bis hin zur Entstehung eines Sonderfonds, der beiden Jugendwerken zur Verfügung stehen würde. Wie in anderen Bereichen folgten den Ideen keine Taten. Die beiden Jugendwerke realisieren primär bilaterale Projekte und die trilaterale Kooperation bleibt eine Nebenaktivität ${ }^{(46)}$.

\section{Hochschulen}

Bildung wurde bereits auf dem Gipfel des Weimarer Dreiecks im Jahre 1998 als Priorität definiert. Dabei rückte die Aufmerksamkeit sowohl des polnischen und des französischen Staatspräsidenten als auch des deutschen Bundeskanzlers auf die Kooperation der Universitäten ins Zentrum. Während des Posener Gipfels wurde die Gründung einer Europäischen Universität in Polen vorgeschlagen. Die Idee wurde aber nicht weiter verfolgt und auf den beiden nächsten Gipfeln in Nancy (1999) und in Neustadt (2001) wurde die Hochschulzusammenarbeit überhaupt nicht erwähnt. Auf dem Gipfel in Wrocław im Jahre 2003 wurde mit dem Umbau der Europa-Universität Viadrina zu einer trilateralen deutsch-französisch-polnischen eine interessante Konzeption entwickelt, die ebenfalls nicht realisiert wurde. Auch wenn auf den nächsten Gipfeln die Idee der verstärkten Kooperation im Hochschulwesen wiederkehrte, wurden konkrete Ergebnisse nicht erreicht. Wie Klaus-Heinrich Standke ausführt, gibt es kein einziges Beispiel der trilateralen deutsch-französisch-polnischen Hochschulkooperation. Die Kooperation erfolgt nur im bilateralen Rahmen. Es ist auch interessant darauf hinzuweisen, dass die für das Hochschulwesen verantwortlichen Minister der drei Länder in den letzten Jahren nicht nur keine gemeinsame Hochschulstrategie entwickelt haben, sondern sich nicht einmal getroffen haben ${ }^{(47)}$.

43 „DPJWeröffnetWartelistefürFörderanträge“,DPJW,http://www.dpjw.org/news-projekte/nachrichten/ dpjw-eroeffnet-eine-warteliste-fuer-die-foerderantraege/ (Zugriff am 10.06.2016).

44 Vgl. DPJW/ PNWM, Daten und Fakten DPJW 2013 in Zahlen und Grafiken, Potsdam/Warschau, Dezember 2014, S. 31-32.

45 Wolfgang Ilg, Judith Duвisкi (Hg.), Begegnung schafft Perspektiven. Empirische Einblicke in internationale Jugendbegegnungen, Berlin/Potsdam/Paris/Warschau, Deutsch-Französisches Jugendwerk und Deutsch-Polnisches Jugendwerk, 2011.

46 Vgl. Kl.-H. StandKe, „Jugendaustausch im Weimarer Dreieck“(Anm. 33), S. 610-612.

47 Vgl. Klaus-Heinrich StAndKe, „Die Rolle der Hochschulen im Weimarer Dreieck“, in: Kl.-H. STANDKE (Hg.), Europa (Anm. 33), S. 685-708; Klaus-Heinrich STAndKe, „Schaffung eines Europäischen Hochschulraums? Das Weimarer Dreieck als Laboratorium für gesamteuropäische Strategien“, Dokumente, Zeitschrift für den deutsch-französischen Dialog, 59/6 (2003), S. 33-37. 
Dagegen bestehen zahlreiche bilaterale Kooperationspartnerschaften. Die Daten des Hochschulkompasses zeigen, dass zwischen Deutschland und Frankreich 2668 und zwischen Deutschland und Polen 1285 Kooperationsvereinbarungen bestehen ${ }^{(48)}$. Als Musterbeispiele der bilateralen Kooperation dienen die Universität des Saarlandes, die 1950 zur „europäischen Universität“ proklamiert wurde, die Europa-Universität Viadrina und das Willi-Brandt-Zentrum an der Universität Wrocław.

Die Analyse der statistischen Daten zeigt, dass die Studentenmobilität zwischen den drei Staaten relativ niedrig ist. Frankreich, Deutschland und Polen gehören in Europa zu der Gruppe der Länder mit der höchsten Zahl an Studenten. Die Zahl der DAADStipendiaten hält sich jedoch in einem überschaubaren Rahmen. Noch deutlicher ist das relativ geringe Interesse der Studenten für andere Staaten des Weimarer Dreiecks an der Mobilität im Rahmen des Erasmus-Programms abzulesen. Nur knapp 12\% der französischen Erasmus-Teilnehmer entscheiden sich für ein Studium in Deutschland, und lediglich über 15\% der deutschen am Erasmus-Programm teilnehmenden Studenten verbringen ein oder zwei Semester in Frankreich. Die Studenten aus beiden Staaten wählen lieber Spanien als Zielland. Für 2,5\% der französischen und der deutschen Studenten, die am Erasmus-Programm teilnehmen, ist Polen das Zielland. Was überraschen kann: der Anteil ist im Fall der französischen Studenten etwas höher.

Tabelle 6. Zahl der Studenten in den Ländern des Weimarer Dreiecks im Jahre 2013, in 1000

\begin{tabular}{|r|r|}
\hline Deutschland & 2780 \\
\hline Frankreich & 2338 \\
\hline Polen & 1902 \\
\hline
\end{tabular}

Quelle: Eurostat, Number of tertiary education students, http://ec.europa.eu/eurostat/statistics-explained/index.php/File:Number_ of_tertiary_education_students,_2013_\%28thousands\%29_ET15.png

Tabelle 7. Erasmusmobilität der Studenten. Akademisches Jahr 2012/2013

\begin{tabular}{|c|c|c|c|c|}
\hline Heimatland & Zielland & $\begin{array}{l}\text { Zahl der Studenten, die } \\
\text { das Zielland wählen }\end{array}$ & $\begin{array}{c}\text { Zahl der Studenten } \\
(100 \%)\end{array}$ & $\begin{array}{c}\text { Anteil des } \\
\text { Ziellandes (in \%) }\end{array}$ \\
\hline Frankreich & Deutschland & 4218 & \multirow[b]{2}{*}{35311} & 11,94 \\
\hline Frankreich & Polen & 895 & & 2,53 \\
\hline Deutschland & Frankreich & 5450 & \multirow[b]{2}{*}{34891} & 15,62 \\
\hline Deutschland & Polen & 874 & & 2,5 \\
\hline Polen & Frankreich & 1191 & \multirow[b]{2}{*}{16221} & 7,34 \\
\hline Polen & Deutschland & 2374 & & 14,63 \\
\hline
\end{tabular}

Quelle: European Commission, Erasmus statistics from 2012-2013.

Annex 2: Erasmus student mobility; home and destination countries.

48 Vgl. Hochschulkompass. Kooperationen nach Staaten, stand 26.03.2016.http://www.hochschul kompass.de/auslandskooperationen/kooperationen-nach-staaten.html (Zugriff am 28.03.2016). 
Tabelle 8. DAAD-Länderstatistik 2014, Geförderte aus dem Ausland (gesamt)

\begin{tabular}{|r|c|c|}
\cline { 2 - 3 } \multicolumn{1}{c|}{} & Frankreich & Polen \\
\hline 1. Individualförderung & 155 & 332 \\
\hline grundständig Studierende & 87 & 178 \\
\hline $\begin{array}{r}\text { Studierende mit erstem Abschluss } \\
\text { (Graduierte) }\end{array}$ & 58 & 102 \\
\hline $\begin{array}{c}\text { Wissenschaftler und Hochschul- } \\
\text { lehrer (inkl. Postdoktoranden) }\end{array}$ & 10 & 52 \\
\hline 2. Projektförderung & 292 & 1093 \\
\hline grundständig Studierende & 126 & 304 \\
\hline $\begin{array}{c}\text { Studierende mit erstem Abschluss } \\
\text { (Graduierte) }\end{array}$ & 97 & 370 \\
\hline $\begin{array}{c}\text { Wissenschaftler und Hochschul- } \\
\text { lehrer (inkl. Postdoktoranden) }\end{array}$ & 69 & 419 \\
\hline 3. EU-Mobilitätsprogramme & 2 & 4 \\
\hline $\begin{array}{c}\text { Wissenschaftler und Hochschul- } \\
\text { lehrer (inkl. Postdoktoranden) }\end{array}$ & 2 & 4 \\
\hline zusammen & 449 & 1429 \\
\hline
\end{tabular}

Quelle: https://www.daad.de/medien/daad-1\%C3\%A4nderstatistik_104_.pdf, https://www.daad.de/medien/daad-1\%C3\%A4nderstatistik_6_.pdf,

Referat Monitoring, Evaluation und Studien - S1, Juli 2015.

\section{Schlussfolgerungen}

Das Weimarer Dreieck hat eine wichtige Rolle in den polnischen Bemühungen um den EU-Beitritt gespielt. Es hat Deutschland und Frankreich geholfen, das Regelwerk der EU Richtung Ost auszudehnen. Heute fehlt aber Frankreich, Deutschland und Polen eine klare Vision, welche Rolle das Weimarer Dreieck spielen soll. Es wuchs in den letzten zwei Jahrzehnten zu einer rhetorischen Figur und seine politische Rolle bleibt unklar. Eine Chance für den Entwurf einer neuen Vision des Weimarer Dreiecks stellte die Kooperation im Bereich Jugendaustausch und Hochschulen dar, deren Erfolg zur Schaffung eines transnationalen öffentlichen Raums im Zentrum Europas führen könnte. Wie die Untersuchung zeigt, konnten die Entscheidungsträger nicht über die konzeptionelle Diskussion hinauskommen und es wurde keine institutionelle Basis geschaffen. Frankreich, Deutschland und Polen setzen jeweils auf bilaterale Zusammenarbeit. Das geringe Interesse an den Sprachen der Partnerländer machte die Entstehung einer trinationalen Öffentlichkeit unmöglich. Auch die Überzeugung, dass das Erlernen der französischen oder deutschen Sprache in der Zukunft vorteilhaft sein kann, ist begrenzt. Das Interesse am Erlernen der polnischen Sprache bleibt komplett außen vor. Dank dem DFJW und dem DPJW, den zwei Säulen dieser Zusammenarbeit, nehmen jährlich mehrere 100000 junge Menschen an den unterschiedlichen Programmen der beiden Jugendwerke teil. Als Studenten wählen sie aber lieber andere Länder, wenn sie am Erasmus- Austauschprogramm teilnehmen. Das Interesse an den anderen Staaten des Weimarer Dreiecks hält also nicht lange. 
Das Weimarer Dreieck ist weiter nur die Summe der bilateralen deutsch-französischen und deutsch-polnischen Beziehungen. Dafür, dass Deutschland eine zentrale Rolle spielt, sind die Gründe vielfältig. Der wichtigste ist wahrscheinlich die wirtschaftlich-politische Bedeutung des Landes in Europa. Der zweite Grund ist die aktive Politik der Bundesregierung. Das Weimarer Dreieck fördert aber das gegenseitige Interesse der Polen an Frankreich und der Franzosen an Polen nicht.

Das Weimarer Dreieck bleibt ein Projekt der politischen Eliten ohne öffentliche Resonanz, eine nicht erfüllte Hoffnung der französisch-deutsch-polnischen Zusammenarbeit. Auf eine deutsch-französisch-polnische Öffentlichkeit werden wir wohl noch lange warten müssen.

\section{Zusammenfassung}

Zusammen mit Frankreich und Polen gründete Deutschland im Jahre 1991 das Weimarer Dreieck. Es sollte als Konsultationsforum der deutschen, französischen und polnischen Regierungen dienen und die drei Gesellschaften einander annähern. Ziel dieses Beitrages ist, die Erfolge auf diesem Weg zu überprüfen. Es wird die These formuliert, dass das Weimarer Dreieck ein Elitenprojekt geblieben ist, ohne große Resonanz in der deutschen, französischen oder polnischen Öffentlichkeit. Als Maßstab wird die deutschfranzösisch-polnische Kooperation im Bereich Jugendaustausch und Hochschulwesen herangezogen. In diesem Beitrag wird eine besondere Aufmerksamkeit der Sprachförderung geschenkt.

\section{Résumé}

L'Allemagne, la France et la Pologne ont créé en 1991 le Triangle de Weimar qui devait servir de forum de consultation des gouvernements allemand, français et polonais et rapprocher les trois sociétés. L'objectif de ce texte est de vérifier la réussite de cette entreprise. Il émet la thèse que le Triangle de Weimar est resté le projet d'une élite sans grande résonance dans les espaces publics allemand, français et polonais. Appuyant son analyse sur la coopération en matière universitaire et d'échanges de jeunes, il accorde une attention particulière à l'enseignement des langues des partenaires.

\footnotetext{
Abstract

In 1991 Germany, together with France and Poland, founded the Weimar Triangle. It was meant to serve as a consultative forum of the German, French and Polish Governments as well as to bring the three societies closer together. The aim of this paper is to review the results of this endeavour. I will argue that the Weimar Triangle has remained a project of elites without substantial resonance in the German, French or Polish public. What I use as a benchmark here is the French-German-Polish cooperation in youth exchange and higher education. Special attention is paid to the promotion of language of the partner countries.
} 